Advances in Geosciences, 4, 1-2, 2005

SRef-ID: 1680-7359/adgeo/2005-4-1

European Geosciences Union

(C) 2005 Author(s). This work is licensed

under a Creative Commons License.

\title{
Preface
}

\section{Model integration and development of modular modelling systems}

\author{
P. Krause, S. Kralisch, and W.-A. Flügel \\ Department for Geoinformatics, Hydrology and Modelling, Friedrich-Schiller-University Jena, Germany
}

\section{Introduction}

Model development and application for Integrated Water Resources Management (IWRM) of river basins is facing the challenge of reflecting and combining approaches from natural as well as social sciences. Modelling techniques and model design differ in these various scientific disciplines; furthermore, they address issues of system complexity and different scales in multiple ways. Integration and coupling of such modelling approaches for IWRM has led to intensified research activities related to the development of generic modelling frameworks and modular modelling systems. The papers in this special issue, which provide an overview of this field of research, represent a selected contribution to a special session of the biannual meeting of Integrated Environmental Software Society (IEMSS) that took place in July 2004 in Osnabrück, Germany. This session focussed on providing a comprehensive overview as well as a discussion platform for recent advances and trends currently under development in this regard.

For integrated studies, modelling approaches from different domains or disciplines have to be coupled or combined. The contributions to this special session show that there are two major tracks of development: (i) the direct coupling of single models, mostly by the definition of interfaces and (ii) the use of modular modelling systems, which are working on process level. Both approaches have specific advantages and disadvantages, which are discussed in detail in various papers of this special issue. A third way for model interaction is to combine models with respect to their spatial representation and distribution by using GIS systems.

\section{Model coupling and integration}

The most straightforward way to combine modelling approaches from different disciplines and domains is the coupling of stand-alone models to provide tools for the assessment of interactions and relationships between different parts of the research field under consideration.

Correspondence to: P. Krause

(p.krause@uni-jena.de)
Different approaches to coupled modelling vary concerning their level of complexity of potential interaction and feedback between single components. Leon et al. as well as Lotze-Campen discuss the simplest way to combine two models by linear coupling, which uses the output of one model as input for another. The technical challenge of such a one-way coupling is the accurate consideration of spatial and temporal resolution of different models, which can be achieved by transferring all models to the same spatial distribution (raster) and temporal resolution. This method, which allows for a mostly independent application of individual models, has the advantage that their established code and design does not have to be altered or adapted. Implemented interfaces take care of suitability and correctness of the data transfer between the models. The disadvantage is that potential interactions and influences between the models can only be considered in one direction.

If more than two models have to be coupled, considerations of the correct order have to be taken into account as well as technical solutions for proper data format and transfer. Therefore, "umbrella" systems, which provide standardised methods and interfaces, proved to be helpful. The Model Integration System LIANA (Hofman), which serves as a container or shell application, is an example of such an "umbrella" system. These systems aid in standardising model responses and transfer of output from one model to input of another; however, they are not able to provide solutions for a more complex consideration of feedback or interactions between the coupled approaches.

The assessment of such interactions necessitates a more sophisticated method working in two directions. A possible solution is the use of predefined tools such as High Level Architecture (HLA) (Lindenschmidt et al.), which allows for a tighter integration of various models by defining interfaces that work in two directions. The authors demonstrate how three submodels for water quality simulations can be combined with HLA in such a way that they are able to interact by exchanging states during the application. This type of model coupling requires more effort regarding the models because they have to be adapted to some extent. However, a larger flexibility is gained, which can be used for more detailed analyses of the coupled models, for instance by uncertainty analyses with Monte Carlo simulations. 
The EU HarmonIT project is the most sophisticated approach for model coupling. Great effort is spent on the development of concepts called Open Modelling Interfaces OpenMI (Gregersen et al. and Blind et al.), which allow the combination of various models. In OpenMI existing models are integrated into model components, which are connected by well-defined interfaces based on a pull driven structure (Gregersen et al.). This approach enables single model components to enquire for required data; furthermore, it allows the system to retrieve this data from other model components. The risk of deadlocks (two or more models are waiting for input from each other) is minimized by enabling model components to produce preliminary estimations of needed data (Blind et al.). In addition to coupling interfaces, OpenIM features generic tools for data visualisation, wrapping of existing legacy code, logging, optimisation etc.

Haas and Jäger present an interesting and cross discipline method for integrating human perception into climatic change risk analyses by using an agent-based approach (a popular modelling method in social sciences) called LAGOM.

\section{Modular modelling systems}

In the last decade, modular modelling systems and frameworks for model integration have been developed to overcome problems of limited flexibility of coupled approaches. The various solutions have in common, that they are focussing more on a process-oriented viewpoint and not so much on the integration or coupling of full models. Therefore, single process descriptions and implementations are extracted from existing models or newly developed to build up module libraries of process components. Additionally, most modular modelling or framework systems provide common components like standardised graphical user interfaces (GUI), interfaces and methods for data in- and output (IO) and data processing as well as tools for model analysis and data visualisation.

Examples for such modular modelling systems presented in this special issue are the Object Modelling System OMS (Ahuja et al. and Kralisch et al.) and DIVA (Hinkel).

The main reason for the development of OMS was the increasing complexity and multidisciplinary nature of problems raised by optimal management of natural resources and the environment (Ahuja et al.). To face these challenges, a new framework for model development and application is required that integrates existing future natural resource models into a common, collaborative and flexible system. The current OMS can be described as a computer framework consisting of a library of science, control and database modules, a model builder, user-friendly interfaces and a model application framework. To transfer existing legacy model code into OMS compliant modules, an automated JAVA wrapper generator for embedding of legacy code components written in Fortran or $\mathrm{C}++$ is also part of the system. A comprehensive description as well as two examples of model implementa- tion in OMS is given by Ahuja et al. Kralisch et al. have developed a slightly different development branch of OMS, in which the basic OMS software has been extended by special components featuring spatial and temporal iterators that provide the software environment for distributed hydrological models. A successful example of the migration of the J2000 model into OMS compliant components and the model application inside OMS is presented in Kralisch et al. This example shows one of the major disadvantages of such complex modelling systems: because of their flexibility, a large amount of overhead is necessary to consider various constraints and requirements, which on the other hand have major influence on the performance of the entire system. Comparisons between the original J2000 and the one embedded into OMS reveal that the OMS version worked 3 to 10 times slower on the same catchment and time series. The transfer of the basic OMS ideas into a faster but less flexible system is currently under development at the FSU Jena.

The development of the second framework system DIVA, presented by Hinkel, was driven by demands of an EU project for which a framework for the integration of different models was needed. In the scope of this project, great attention was paid to the problem of how modelling approaches can be modularized and which side effects and interfaces have to be considered. The development of DIVA (Hinkel) was guided by a four-step concept breaking the model development into (1) the ontology (often also called perception), (2) the mathematical problem, (3) the algorithm(s) and (4) the computer model.

\section{GIS integration}

GIS are often used during the pre- and post-processing of continuous models to address requirements of distributed assessment and decision making on complex natural and human influenced systems. However, the integration of temporal dynamics directly into GIS based software is currently still a problem. In this issue, Pullar and Matejicek present two solutions for this problem.

Pullar presents a generic environmental modelling language integrated into a GIS, which supports spatial-temporal operators to model physical interactions either in an isolated location or as interactions propagating spatially across landscape surfaces.

Matejicek used ArcGIS and ArcView programming tools for the development of spatial and temporal modelling of air pollution in urban areas. In the case study, various spatial GIS data layers were combined with relational data stored in RDBMS to provide input for the spatial and temporal modelling.

Acknowledgements. The session organisers like to thank all the authors for the great cooperation during the meeting in Osnabrück and the peer review and preparation of the manuscripts for this special issue. A special thank goes to Nadine Deisel from COPERNICUS for putting the pieces together. 